\title{
INVARIANT AFFINE CONNECTIONS ON LIE GROUPS
}

\author{
H. TURNER LAQUER
}

\begin{abstract}
The space of bi-invariant affine connections is determined for arbitrary compact Lie groups. In particular, there is a surprising new family of such connections on $S U(n)$.
\end{abstract}

\section{INTRODUCTION}

Invariant connections on homogeneous spaces have been well understood for years but much of the interest ceased after consideration of the canonical connection in general and the Levi-Civita connection in the case of the frame bundle [5]. The main objective of this paper is to give a complete description of the bi-invariant affine connections on compact Lie groups, i.e., those connections in the frame bundle which are invariant under both left and right translations. A sequel to this paper will extend these results to arbitrary compact irreducible Riemannian symmetric spaces [8].

The first five sections of this paper describe invariant connections in general and affine connections in particular from the point of view of homogeneous principal bundles. These are essentially those principal bundles over homogeneous spaces which admit a lifting of the group action on the base manifold to give a fiber transitive action on the total space. This categorical viewpoint is quite useful for dealing with different descriptions of isomorphic objects. It helps separate the ideas of homogeneity from actual constructions involving Lie groups. The main result in these sections is Theorem 5.1 which gives several equivalent descriptions of the set of invariant affine connections on a homogeneous space.

Sections 6 through 10 deal with the determination of all bi-invariant affine connections on compact Lie groups. Theorem 8.1 gives a complete classification of such connections in the compact simple case. This theorem was essentially the result of a case by case computation using representation theory. The surprising result is that while in most cases the only such connections are from a well-known one-dimensional family (containing the canonical, (+)-, and (-)connections), in the case of $S U(n)$ there is a second family of such connections. These results are then extended in Theorem 9.1, where the dimension of the space of bi-invariant affine connections is determined for arbitrary compact Lie groups. Finally, $\S 10$ includes a more detailed description of these connections

Received by the editors July 21, 1987 and, in revised form, June 25, 1990.

1991 Mathematics Subject Classification. Primary 53C05; Secondary 53C30, 22 E15.

Key words and phrases. Affine, connection, invariant, homogeneous principal bundle, Lie group. 
in the cases of $S U(n)$ and $U(n)$. The six-dimensional space of such connections on $U(n)$ is also quite interesting. In both cases, the extra connections are related to the bilinear operation $\theta(x, y)=i \cdot(x \cdot y+y \cdot x)$ on the Lie algebra $\mathfrak{u}(n)$.

\section{HOMOGENEOUS PRINCIPAL BUNDLES}

A homogeneous principal bundle is a triple $(G, P(M, U, \pi), \alpha)$, where $G$ is a Lie group, $\pi: P \rightarrow M$ is a principal $U$-bundle over a homogeneous space $M$, and $\alpha$ is a homogeneous $G$-action on $P$, i.e., there must be smooth group actions $\alpha_{P}: G \times P \rightarrow P$ and $\alpha_{M}: G \times M \rightarrow M$, written multiplicatively, with $\alpha_{M}$ transitive and such that the compatibility condition $\pi(g \cdot p)=g \cdot \pi(p)$ holds. In addition, the left $G$-action and the right $U$-action on $P$ must commute, i.e., $(g \cdot p) \cdot u=g \cdot(p \cdot u)$.

The homogeneous principal bundles form a category, denoted HPB, by letting a morphism $\phi:\left(G_{1}, P_{1}\left(M_{1}, U_{1}, \pi_{1}\right), \alpha_{1}\right) \rightarrow\left(G_{2}, P_{2}\left(M_{2}, U_{2}, \pi_{2}\right), \alpha_{2}\right)$ consist of differentiable maps $\phi_{P}: P_{1} \rightarrow P_{2}$ and $\phi_{M}: M_{1} \rightarrow M_{2}$ and Lie group homomorphisms $\phi_{G}: G_{1} \rightarrow G_{2}$ and $\phi_{U}: U_{1} \rightarrow U_{2}$ satisfying the following conditions:

(i) $\pi_{2} \circ \phi_{P}=\phi_{M} \circ \pi_{1}$

(ii) $\phi_{P}(p \cdot u)=\phi_{P}(p) \cdot \phi_{U}(u)$,

(iii) $\phi_{P}(g \cdot p)=\phi_{G}(g) \cdot \phi_{P}(p)$.

Note, it then follows that

(iv) $\phi_{M}(g \cdot m)=\phi_{G}(g) \cdot \phi_{M}(m)$.

Let $\mathrm{HPB}_{0}$ denote the category whose objects are homogeneous principal bundles with base points $-\left(G, P(M, U, \pi), \alpha, p_{0}\right)$, where $p_{0}$ is some point in the total space $P$ - and in which morphisms must preserve base points. Let $\mathrm{HS}$ and $\mathrm{HS}_{0}$ be the related categories of homogeneous spaces - with objects $(G, M, \alpha)$ and $\left(G, M, \alpha, m_{0}\right)$ and in which morphisms $\phi=\left(\phi_{G}, \phi_{M}\right)$ must satisfy condition (iv).

As with homogeneous spaces where the choice of base point gives a correspondence to quotient manifolds, it is the category $\mathrm{HPB}_{0}$ which is more closely related to standard constructions involving Lie groups. Given $(G, P(M, U, \pi)$, $\left.\alpha, p_{0}\right) \in \mathrm{HPB}_{0}$, let $H$ be the isotropy subgroup at $m_{0}=\pi\left(p_{0}\right)$. Define a homomorphism $\lambda: H \rightarrow U$ by letting $h \cdot p_{0}=p_{0} \cdot \lambda(h)$ and let $P_{\lambda}$ be the principal bundle (cf. $[5,6,7])$

$$
P_{\lambda}=G \times{ }_{H} U=G \times U /(g h, u) \sim(g, \lambda(h) u) .
$$

This principal $U$-bundle over $G / H$ has a standard homogeneous $G$-action defined by $g_{1} \cdot[g, u]=\left[g_{1} \cdot g, u\right]$ and the original bundle is isomorphic in $\mathrm{HPB}_{0}$ to the bundle $\left(G, P_{\lambda}(G / H, U, \pi), \alpha,[e, e]\right)$. So up to isomorphism in $\mathrm{HPB}_{0}$, it is the "structure homomorphism" $\lambda$ which really determines the homogeneous principal bundle.

\section{THE FRAME BUNDLE AS A HOMOGENEOUS PRINCIPAL BUNDLE}

The frame bundle of a homogeneous space $\left(G, M, \alpha, m_{0}\right)$ can be viewed as a homogeneous principal bundle in a variety of ways. Clearly, it is the differential of left translation $\left(T L_{g}\right)$ which provides the left $G$-action on the total space. However, the structure group can change, depending on what is meant 
by a "frame", and in addition, different base points give different structure homomorphisms.

A frame $p$ at a point $m \in M$ can be viewed as a real linear isomorphism $p: V \rightarrow T_{m} M$, where $V$ is some "model" vector space. The set of such frames becomes a homogeneous principal $\operatorname{Aut}(V)$-bundle by letting

$$
g \cdot p \cdot u=T L_{g} \circ p \circ u \text {. }
$$

Clearly this action is compatible with the given action on the homogeneous space $M$. A base point is then some fixed isomorphism $A: V \rightarrow T_{m_{0}} M$. If $W$ is some other model and if $B: W \rightarrow T_{m_{0}} M$ is a base point in the corresponding homogeneous principal bundle, then the two bundles are naturally isomorphic in $\mathrm{HPB}_{0}$. Indeed, the mappings $\phi_{G}(g)=g, \phi_{M}(m)=m, \phi_{P}(p)=p \circ A^{-1} \circ B$, and $\phi_{U}(u)=B^{-1} \circ A \circ u \circ A^{-1} \circ B$ give an explicit $\mathrm{HPB}_{0}$-isomorphism between them.

There are three models which arise naturally in this context. The model $V=$ $\mathbb{R}^{n}$ (where $n$ is the dimension of $M$ ) corresponds to the ordered basis viewpoint of frames (by looking at the image under $p$ of the standard basis of $\mathbb{R}^{n}$ ). In this case, a base point is the choice of a distinguished basis $\left(E_{1}, \ldots, E_{n}\right)$ for $T_{m_{0}} M$. The resulting structure homomorphism $\lambda: H \rightarrow G L(n, \mathbb{R})$ is the isotropy representation

$$
T L_{h}\left(E_{i}\right)=E_{j} \cdot(\lambda(h))_{i}^{j} .
$$

A second model is given by $V=T_{m_{0}} M$. In this case the natural base point is the identity isomorphism $V \rightarrow T_{m_{0}} M$. The resulting structure homomorphism is the differential of left translation $T L: H \rightarrow \operatorname{Aut}\left(T_{m_{0}} M\right)$. Finally, if the homogeneous space is reductive, i.e., if the Lie algebra $\mathfrak{g}$ can be expressed as a direct sum $\mathfrak{h} \oplus \mathfrak{m}$ with $\operatorname{Ad}(H) \mathfrak{m} \subseteq \mathfrak{m}$, then the subspace $\mathfrak{m}$ can serve as the model. In this case the differential at the identity of the mapping $g \mapsto g \cdot m_{0}$ restricts to give an isomorphism $\mathfrak{m} \subseteq \mathfrak{g}=T_{e} G \rightarrow T_{m_{0}} M$. This is the natural choice of base point for this version of the frame bundle. The corresponding structure homomorphism is then given by $\operatorname{Ad}: H \rightarrow \operatorname{Aut}(\mathfrak{m})$.

\section{INVARIANT CONNECTIONS AND WANG'S THEOREM}

By definition, a connection $A$ in a principal bundle $P(M, U, \pi)$ is a smoothly varying choice of horizontal subspaces $\mathscr{H}_{p}{ }^{A} \subseteq T_{p} P$. These subspaces must be complementary to the vertical subspaces $\mathscr{V}_{p}=\operatorname{kernel}(T \pi)$ and they must be invariant under the right action of $U$ on $P$, i.e. $\mathscr{H}_{p \cdot u}^{A}=T R_{u} \mathscr{H}_{p}^{A}$. Homogeneous principal bundles can have homogeneous or G-invariant connections in which the horizontal subspaces are also left $G$-invariant, i.e. $\mathscr{H}_{g}{ }^{A} p=T L_{g} \mathscr{H}_{p}{ }^{A}$. The set of such $G$-invariant connections will be denoted $\mathscr{A}_{G}(P)$.

Wang's theorem [11] describes the set of $G$-invariant connections in the bundle $P_{\lambda}$. Let $\mathfrak{g}, \mathfrak{h}$, and $\mathfrak{u}$ be the Lie algebras of $G, H$, and $U$ respectively and let $T \lambda: \mathfrak{h} \rightarrow \mathfrak{u}$ be the differential of $\lambda$. Then the invariant connections in $P_{\lambda}$ are in one-to-one correspondence with linear mappings $\xi: \mathfrak{g} \rightarrow \mathfrak{u}$ satisfying the following two conditions:

(i) $\xi(x)=T \lambda(x) \forall x \in \mathfrak{h}$,

(ii) $\xi\left(\operatorname{Ad}_{h} x\right)=\operatorname{Ad}_{\lambda(h)} \xi(x) \forall x \in \mathfrak{g}, h \in H$.

In this description, the set of all vectors of the form $x \oplus-\xi(x) \in \mathfrak{g} \oplus \mathfrak{u}$ gives the horizontal subspace at $(e, e)$ of the connection pulled back to the trivial bundle 
$G \times U \rightarrow G$. Equivalently, if $\rho: G \times U \rightarrow P_{\lambda}$ is the mapping $(g, u) \mapsto[g, u]$ then $y=-\xi(x)$ if and only if $T \rho(x \oplus y) \in \mathscr{X}_{[e, e]}^{\xi}$. Note that this description of invariant connections in terms of linear maps $\xi: \mathfrak{g} \rightarrow \mathfrak{u}$ remains valid for arbitrary bundles in $\mathrm{HPB}_{0}$ - recall that the choice of base point defines the subgroup $H$ and the homomorphism $\lambda: H \rightarrow U$ canonically.

\section{Pullback and association in the category hPB}

Two standard constructions involving principal bundles (pullback and association) carry over to the category of homogeneous principal bundles. If $\left(G_{2}, P_{2}\left(M_{2}, U_{2}, \pi_{2}\right), \alpha_{2}\right) \in \mathrm{HPB}$ and if $\phi:\left(G_{1}, M_{1}, \alpha_{1}\right) \rightarrow\left(G_{2}, M_{2}, \alpha_{2}\right)$ is an HS-morphism then the pullback bundle

$$
\phi^{*}\left(P_{2}\right)=\left\{(m, p) \in M_{1} \times P_{2} \mid \phi_{M}(m)=\pi_{2}(p)\right\}
$$

has a homogeneous $G_{1}$-action given by $g \cdot(m, p)=\left(g \cdot m, \phi_{G}(g) \cdot p\right)$. In addition, $\phi$ extends to give an HPB-morphism $\phi^{*}\left(P_{2}\right) \rightarrow P_{2}$ by letting $\phi_{P}(m, p)=$ $p$ and $\phi_{U}(u)=u$. More generally, if

$$
\phi:\left(G_{1}, P_{1}\left(M_{1}, U_{1}, \pi_{1}\right), \alpha_{1}\right) \rightarrow\left(G_{2}, P_{2}\left(M_{2}, U_{2}, \pi_{2}\right), \alpha_{2}\right)
$$

is an HPB-morphism with $\phi_{U}$ an isomorphism then $P_{1}$ and $\phi^{*}\left(P_{2}\right)$ are isomorphic in HPB. In addition, the following proposition shows that invariant connections can be pulled back under such mappings.

Proposition 4.1. If

$$
\phi:\left(G_{1}, P_{1}\left(M_{1}, U_{1}, \pi_{1}\right), \alpha_{1}, p_{1}\right) \rightarrow\left(G_{2}, P_{2}\left(M_{2}, U_{2}, \pi_{2}\right), \alpha_{2}, p_{2}\right)
$$

is an HPB-morphism with $\phi_{U}$ an isomorphism then there is an induced mapping $\phi^{*}: \mathscr{A}_{G_{2}}\left(P_{2}\right) \rightarrow \mathscr{A}_{G_{1}}\left(P_{1}\right)$ defined by

$$
\mathscr{H}_{p}^{\phi^{*}(A)}=\left\{x \in T_{p} P_{1} \mid T \phi_{P}(x) \in \mathscr{H}_{\phi_{P}(p)}^{A}\right\} .
$$

The mapping $\phi^{*}$ is injective if the tangent mapping $T \phi_{G}: \mathfrak{g}_{1} \rightarrow \mathfrak{g}_{2}$ of Lie algebras is surjective. If the morphism $\phi$ is $H P B_{0}$ then $\phi^{*}$ is also given by

$$
\phi^{*}(\xi)=T \phi_{U}^{-1} \circ \xi \circ T \phi_{G}: \mathfrak{g}_{1} \rightarrow \mathfrak{u}_{1} .
$$

Proof. The fact that this gives a well-defined connection in $P_{1}$ follows from Proposition II.6.2 in [5]. What needs to be proven is that the induced connection is $G_{1}$-invariant and for that it suffices to show $T L_{g} \mathscr{H}_{p}^{\phi^{*}(A)} \subseteq \mathscr{H}_{g \cdot p}^{\phi^{*}(A)}$. Let $x \in \mathscr{H}_{p}^{\phi^{*}(A)}$. Then by property (iii) for HPB-morphisms, it follows that

$$
T \phi_{P} T L_{g}(x)=T L_{\phi_{G}(g)} T \phi_{P}(x) \in T L_{\phi_{G}(g)} \mathscr{H}_{\phi_{P}(p)}^{A}=\mathscr{H}_{\phi_{P}(g \cdot p)}^{A}
$$

and thus the connection $\phi^{*}(A) \in \mathscr{A}_{G_{1}}\left(P_{1}\right)$. If $T \phi_{G}$ is surjective then the mapping $T \phi_{P}: T_{p} P_{1} \rightarrow T_{\phi_{P}(p)} P_{2}$ is surjective for all $p \in P_{1}$. This is clear because $\phi_{U}$ is an isomorphism and because the combination of the left $G$ action and the right $U$-action on a homogeneous principal bundle is transitive. So the horizontal subspaces of the original connection $A$ can be recovered from the horizontal subspaces of the induced connection $\phi^{*}(A)$ and the mapping $\phi^{*}$ is injective. As for the description of $\phi^{*}$ in terms of the linear maps $\xi: \mathfrak{g} \rightarrow \mathfrak{u}$, let $\rho_{i}: G_{i} \times U_{i} \rightarrow P_{i}$ for $i=1,2$ be defined by $\rho_{i}(g, u)=g \cdot p_{i} \cdot u$ and let $\tilde{\phi}(g, u)=\left(\phi_{G}(g), \phi_{U}(u)\right): G_{1} \times U_{1} \rightarrow G_{2} \times U_{2}$. 
Now $y=-\left(\phi^{*}(\xi)\right)(x)$ if and only if $T \rho_{1}(x \oplus y) \in \mathscr{H}_{p_{1}}^{\phi^{*}(\xi)}$, i.e., if and only if $T \phi_{P} T \rho_{1}(x \oplus y) \in \mathscr{H}_{p_{2}}^{\xi}$. Since $\phi_{P} \circ \rho_{1}=\rho_{2} \circ \tilde{\phi}$, this is true if and only if $T \tilde{\phi}(x \oplus y)=z \oplus-\xi(z)$ for some $z \in \mathfrak{g}_{2}$. This implies $-\xi\left(T \phi_{G}(x)\right)=T \phi_{U}(y)$ or equivalently $\phi^{*}(\xi)=T \phi_{U}^{-1} \circ \xi \circ T \phi_{G}$.

The process of forming associated principal bundles also carries over to the homogeneous category. If $\left(G_{1}, P_{1}\left(M_{1}, U_{1}, \pi_{1}\right), \alpha_{1}\right) \in \mathrm{HPB}$ and if $\mu: U_{1} \rightarrow$ $U_{2}$ is a Lie group homomorphism then the associated principal $U_{2}$-bundle

$$
P_{1} \times U_{1} U_{2}=P_{1} \times U_{2} /\left(p \cdot u_{1}, u_{2}\right) \sim\left(p, \mu\left(u_{1}\right) \cdot u_{2}\right)
$$

has a homogeneous $G_{1}$-action given by $g \cdot[p, u]=[g \cdot p, u]$. In addition, the mappings $\phi_{P}(p)=[p, \epsilon], \phi_{M}(m)=m, \phi_{G}(g)=g$, and $\phi_{U}(u)=\mu(u)$ define an HPB-morphism $P_{1} \rightarrow P_{1} \times_{U_{1}} U_{2}$. More generally, if $\phi:\left(G_{1}, P_{1}\left(M_{1}, U_{1}, \pi_{1}\right)\right.$, $\left.\alpha_{1}\right) \rightarrow\left(G_{2}, P_{2}\left(M_{2}, U_{2}, \pi_{2}\right), \alpha_{2}\right)$ is an HPB-morphism for which the induced map $\left(\phi_{G}, \phi_{M}\right)$ on the base manifolds is an HS-isomorphism then the bundle $P_{2}$ is isomorphic in HPB to the associated bundle defined by the homomorphism $\mu=\phi_{U}: U_{1} \rightarrow U_{2}$. In addition, the following proposition shows that invariant connections move forward under such mappings.

Proposition 4.2. If

$$
\phi:\left(G_{1}, P_{1}\left(M_{1}, U_{1}, \pi_{1}\right), \alpha_{1}, p_{1}\right) \rightarrow\left(G_{2}, P_{2}\left(M_{2}, U_{2}, \pi_{2}\right), \alpha_{2}, p_{2}\right)
$$

is an HPB-morphism for which the induced map $\left(\phi_{G}, \phi_{M}\right)$ of base manifolds is an HS-isomorphism then there is an induced map $\phi_{*}: \mathscr{A}_{G_{1}}\left(P_{1}\right) \rightarrow \mathscr{A}_{G_{2}}\left(P_{2}\right)$ defined by

$$
\mathscr{H}_{\phi_{P}(p) \cdot u}^{\phi_{*}(A)}=T R_{u} T \phi_{P} \mathscr{H}_{p}^{A} .
$$

The mapping $\phi_{*}$ is injective if the tangent mapping $T \phi_{U}: \mathfrak{u}_{1} \rightarrow \mathfrak{u}_{2}$ of Lie algebras is injective. If the morphism $\phi$ is $H P B_{0}$ then $\phi_{*}$ is also given by

$$
\phi_{*}(\xi)=T \phi_{U} \circ \xi \circ T \phi_{G}^{-1}: \mathfrak{g}_{2} \rightarrow \mathfrak{u}_{2} .
$$

Proof. The fact that this gives a well-defined connection in $P_{2}$ follows from Proposition II.6.1 in [5]. What needs to be proven is that the induced connection is $G_{2}$-invariant. Property (iii) for HPB-morphisms implies $L_{\phi_{G}(g)} \circ R_{u} \circ \phi_{P}=$ $R_{u} \circ \phi_{P} \circ L_{g}$. This gives

$$
T L_{\phi_{G}(g)} \mathscr{H}_{\phi_{P}(p) \cdot u}^{\phi_{*}(A)}=T R_{u} T \phi_{P} T L_{g} \mathscr{H}_{p}^{A}=\mathscr{H}_{\phi_{P}(g \cdot p) \cdot u}^{\phi_{*}(A)}=\mathscr{H}_{\phi_{G}(g) \cdot \phi_{P}(p) \cdot u}^{\phi_{*}(A)} .
$$

Since the $\phi_{G}$ in an HS-isomorphism $\left(\phi_{G}, \phi_{M}\right)$ must be a Lie group isomorphism, it follows that the connection $\phi_{*}(A) \in \mathscr{A}_{G_{2}}\left(P_{2}\right)$. If $T \phi_{U}$ is injective then $T R_{u} \circ T \phi_{P}: T_{p} P_{1} \rightarrow T_{\phi_{P}(p) \cdot u} P_{2}$ is injective for all $p \in P_{1}$ and $u \in U_{2}$ and thus $\phi_{*}$ is injective. This uses the assumption that $\left(\phi_{G}, \phi_{M}\right)$ is an HS-isomorphism so that tangent vectors corresponding to the action of $G_{1}$ are not annihilated by $T \phi_{P}$. As for the description of $\phi_{*}$ in terms of the linear maps $\xi: \mathfrak{g} \rightarrow \mathfrak{u}$, let $\rho_{1}$ and $\rho_{2}$ be as in the proof of Proposition 4.1. Then

$$
\mathscr{H}_{p_{2}}^{\phi_{*}(\xi)}=T \phi_{P} T \rho_{1}\{x \oplus-\xi(x)\}=T \rho_{2}\left\{T \phi_{G}(x) \oplus-T \phi_{U}(\xi(x))\right\} .
$$

Since $T \phi_{G}$ is an isomorphism, this implies $\phi_{*}(\xi)=T \phi_{U} \circ \xi \circ T \phi_{G}^{-1}$.

\section{INVARIANT AFFINE CONNECTIONS}

An invariant affine connection on a homogeneous space $(G, M, \alpha)$ is a connection in the frame bundle which is also $G$-invariant. By applying the results 
in $\S 3$ to the different models of the frame bundle in $\S 2$, several descriptions of the space of invariant affine connections can be obtained. Specifically, there is the following (cf. [10]).

Theorem 5.1. Let $\left(G, M, \alpha, m_{0}\right)$ be a reductive homogeneous space with reductive decomposition $\mathfrak{g}=\mathfrak{h} \oplus \mathfrak{m}$, where $\operatorname{Ad}(H) \mathfrak{m} \subseteq \mathfrak{m}$. Then the set $\mathscr{A}_{G}(\operatorname{Fr}(M))$ of invariant affine connections is given by any of the following six equivalent descriptions:

(i) $\left\{\xi: \mathfrak{g} \rightarrow \mathfrak{g l}(n, \mathbb{R}) \mid \xi\right.$ linear, $\left.\left.\xi\right|_{\mathfrak{h}}=T \lambda, \xi\left(\operatorname{Ad}_{h} x\right)=\lambda(h) \xi(x) \lambda(h)^{-1}\right\}$,

(ii) $\left\{\xi: \mathfrak{g} \rightarrow \operatorname{End}(\mathfrak{m}) \mid \xi\right.$ linear, $\left.\left.\xi\right|_{\mathfrak{h}}=\mathrm{ad}, \xi\left(\operatorname{Ad}_{h} x\right)=\operatorname{Ad}_{h} \circ \xi(x) \circ \operatorname{Ad}_{h^{-1}}\right\}$,

(iii) $\left\{\xi: \mathfrak{m} \rightarrow \operatorname{End}(\mathfrak{m}) \mid \xi\right.$ linear, $\left.\xi\left(\operatorname{Ad}_{h} x\right)=\operatorname{Ad}_{h} \circ \xi(x) \circ \operatorname{Ad}_{h^{-1}}\right\}$,

(iv) $\left\{\eta: \mathfrak{m} \times \mathfrak{m} \rightarrow \mathfrak{m} \mid \eta\right.$ bilinear, $\left.\eta\left(\operatorname{Ad}_{h} x, \operatorname{Ad}_{h} y\right)=\operatorname{Ad}_{h} \eta(x, y)\right\}$,

(v) $\left\{\eta: T_{m_{0}} \times T_{m_{0}} \rightarrow T_{m_{0}} \mid \eta\right.$ bilinear, $\left.\eta\left(T L_{h} x, T L_{h} y\right)=T L_{h} \eta(x, y)\right\}$,

(vi) $\operatorname{Hom}_{H}^{\mathbb{R}}(\mathfrak{m} \otimes \mathfrak{m}, \mathfrak{m})$.

Proof. Descriptions (i) and (ii) are immediate applications of Wang's theorem to the frame bundle - modeled on $\mathbb{R}^{n}$ in (i) and on $\mathfrak{m}$ in (ii). Note, in (i), that $\lambda$ is the isotropy representation described in $\S 2$. The $\xi$ in (iii) is just the restriction to $\mathfrak{m}$ of the $\xi$ in (ii). Conversely, any $\xi: \mathfrak{m} \rightarrow \operatorname{End}(\mathfrak{m})$ satisfying the conditions in (iii) can be extended to all of $\mathfrak{g}$ by linearity and by requiring $\left.\xi\right|_{\mathfrak{h}}=$ ad . The correspondence between (iii) and (iv) is given by $\xi(x)(y)=\eta(x, y)$. It is easy to see that the conditions in (iii) and (iv) are equivalent. Next, the equivalence between (iv) and ( $v$ ) comes from the canonical isomorphism between $m$ and $T_{m_{0}} M$ given by the differential at the identity of the mapping $g \mapsto g \cdot m_{0}$. Finally, description (vi) comes from (iv) and the universal mapping property for tensor product. Any bilinear map $\eta: \mathfrak{m} \times \mathfrak{m} \rightarrow \mathfrak{m}$ defines a unique linear map $\tilde{\eta}: \mathfrak{m} \otimes \mathfrak{m} \rightarrow \mathfrak{m}$ satisfying $\tilde{\eta}(x \otimes y)=\eta(x, y) \quad \forall x, y \in \mathfrak{m}$. The condition that $\eta$ define an invariant affine connection on $G / H$ is equivalent to the requirement that $\tilde{\eta}$ intertwine the actions of $H$ on $\mathfrak{m} \otimes \mathfrak{m}$ and $\mathfrak{m}$.

The frame bundle of a homogeneous space $\left(G, M, \alpha, m_{0}\right)$ is associated to the canonical bundle $(G, G(M, H, \pi), \alpha, e)$ by the $\mathrm{HPB}_{0}$-morphism $\phi_{P}(g)=$ $T L_{g} \circ T \pi: \mathfrak{m} \rightarrow T_{g} \cdot m_{0} M, \phi_{M}(m)=m, \phi_{G}(g)=g, \phi_{U}(h)=$ Ad $_{h}$. By Wang's theorem invariant connections in the canonical bundle are given by linear mappings $\zeta: \mathfrak{m} \rightarrow \mathfrak{h}$ satisfying $\zeta\left(\operatorname{Ad}_{h} x\right)=\operatorname{Ad}_{h} \zeta(x)$. By Proposition 4.2, any such $\zeta$ gives an invariant affine connection $\xi=\phi_{*}(\zeta)=$ ad $\circ \zeta$. Alternatively, this invariant affine connection is given by $\eta(x, y)=[\zeta(x), y]$. This mapping of connections is injective if the representation ad: $\mathfrak{h} \rightarrow \operatorname{End}(\mathfrak{m})$ is faithful. In the reductive case, as is being assumed here, this will be true if the action of $G$ on $M$ is almost effective.

\section{INVARIANT AFFINE CONNECTIONS ON LIE GROUPS}

The group action of a Lie group $G$ allows the group to be viewed as a homogeneous space in several ways. If the action under consideration is left translation, then the isotropy subgroup is trivial and the corresponding "left invariant" affine connections are given by arbitrary bilinear maps $\eta: \mathfrak{g} \times \mathfrak{g} \rightarrow \mathfrak{g}-$ Theorem 5.1 gives no restriction in this case. A more interesting action is given by a combination of left and right translation. This amounts to viewing $G$ as a homogeneous space for the group $G \times G$, the precise action being 
$\alpha_{M}\left(\left(g_{1}, g_{2}\right), g\right)=g_{1} \cdot g \cdot g_{2}^{-1}$. The resulting invariant affine connections will then be "bi-invariant," i.e., invariant under both the left and right actions of the Lie group $G$.

Theorem 6.1. The space of bi-invariant affine connections on a Lie group $G$ is given by

$$
\left\{\eta: \mathfrak{g} \times \mathfrak{g} \rightarrow \mathfrak{g} \mid \eta \text { bilinear, } \eta\left(\operatorname{Ad}_{g} x, \operatorname{Ad}_{g} y\right)=\operatorname{Ad}_{g} \eta(x, y) \forall g \in G\right\}
$$

or, equivalently, by the space $\operatorname{Hom}_{G}^{\mathbb{R}}(\mathfrak{g} \otimes \mathfrak{g}, \mathfrak{g})$.

Proof. The isotropy subgroup for the action of $G \times G$ is the diagonal subgroup $\Delta=\{(g, g)\}$. The Lie algebra of $\Delta$ is $\mathfrak{h}=\{(x, x) \in \mathfrak{g} \oplus \mathfrak{g}\}$ and the reductive complement $\mathfrak{m}=\{(x,-x) \in \mathfrak{g} \oplus \mathfrak{g}\}$. Thus $\mathfrak{h}$ and $\mathfrak{m}$ are both isomorphic to $\mathfrak{g}$ as $\Delta(=G)$-modules. The theorem then follows immediately from Theorem 5.1 .

When the Lie group $G$ is compact and simple the canonical bundle $G \times$ $G \rightarrow G$ has a one-dimensional family of invariant connections corresponding to the mappings $\zeta(x,-x)=(\mu x, \mu x): \mathfrak{m} \rightarrow \mathfrak{h}$ for $\mu \in \mathbb{R}$. Cases $\mu=0,1$, and -1 correspond to the so-called canonical, (+)-, and (-)-connections on a Lie group. The corresponding invariant affine connections are then given by $\eta(x, y)=\mu \cdot[x, y]: \mathfrak{g} \times \mathfrak{g} \rightarrow \mathfrak{g}$. In $\S 8$ it will be proven that these are the only invariant affine connections in all cases except for $S U(n)$ for $n \geq 3$.

\section{CASIMIR OPERATORS AND A COMPUTATIONAL LEMMA}

Given a representation $\rho: G \rightarrow \operatorname{Aut}(V)$, the Casimir operator of $V$ is defined by $\Gamma(V)=\sum\left(T \rho\left(e_{i}\right)\right)^{2}$, where $\left\{e_{i}\right\}$ is an orthonormal basis for the Lie algebra $\mathfrak{g}$. This depends on the choice of an inner product on $\mathfrak{g}$ but not on the choice of basis. If $G$ is compact and simple, then a natural inner product on $\mathfrak{g}$ is provided by the negative of the Killing form $B_{\mathfrak{g}}(x, y)=\operatorname{trace}\left(\operatorname{ad}_{x} \operatorname{ad}_{y}\right)$, but in general, any ad-invariant inner product $(\langle[x, y], z\rangle=\langle x,[y, z]\rangle)$ can be used. If $V$ is irreducible then $\Gamma(V)$ will be a negative or zero scalar. The following lemma will be needed for the computations in $\S 8$.

Lemma 7.1. Suppose $\mathfrak{g}$ is simple and let $V \subseteq \Lambda^{2} \mathfrak{g}$ be the kernel of the natural map $\wedge^{2} \mathfrak{g} \rightarrow \mathfrak{g}$ induced by the alternating bilinear map $(x, y) \mapsto[x, y]: \mathfrak{g} \times \mathfrak{g} \rightarrow$ $\mathfrak{g}$. Then $V$ is a $G$-module and the Casimir operator of $V$ is exactly twice that of the adjoint representation $\mathfrak{g}$.

Proof. Since $\operatorname{Ad}_{g}[x, y]=\left[\operatorname{Ad}_{g} x, \operatorname{Ad}_{g} y\right]$ the natural map $\wedge^{2} \mathfrak{g} \rightarrow \mathfrak{g}$ is a $G$ module map and thus $V$ is $G$-invariant. Let $\left\{e_{i}\right\}$ be an orthonormal basis for $\mathfrak{g}$ and let $\left[e_{i}, e_{j}\right]=C_{i j}^{k} e_{k}$. Because the basis is orthonormal relative to an ad-invariant inner product, the structure constants $C_{i j}^{k}$ have a cyclic symmetry in $i, j$, and $k$ along with the usual skew-symmetry. In addition, the Jacobi identity implies

$$
C_{j m}^{i} C_{n i}^{k}=-C_{m n}^{i} C_{j i}^{k}-C_{n j}^{i} C_{m i}^{k}
$$

A general element of $\wedge^{2} \mathfrak{g}$ is given by $a^{j k} e_{j} \wedge e_{k}$, where the summation goes over all $j$ and $k$ by assuming $a^{j k}=-a^{k j}$. Suppose $a^{j k} e_{j} \wedge e_{k} \in V$, i.e., 


$$
\begin{aligned}
& a^{j k} C_{j k}^{i} e_{i}=0 . \text { Then } \\
& \begin{aligned}
\sum( & \left.\rho\left(e_{i}\right)\right)^{2} a^{j k} e_{j} \wedge e_{k}=a^{j k} \rho\left(e_{i}\right)\left(\left[e_{i}, e_{j}\right] \wedge e_{k}+e_{j} \wedge\left[e_{i}, e_{k}\right]\right) \\
& =a^{j k}\left[e_{i},\left[e_{i}, e_{j}\right]\right] \wedge e_{k}+a^{j k} e_{j} \wedge\left[e_{i},\left[e_{i}, e_{k}\right]\right]+2 \cdot a^{j k}\left[e_{i}, e_{j}\right] \wedge\left[e_{i}, e_{k}\right] \\
& =2 \cdot \Gamma(\mathfrak{g}) \cdot\left(a^{j k} e_{j} \wedge e_{k}\right) .
\end{aligned}
\end{aligned}
$$

This follows because

$$
\left[e_{i},\left[e_{i}, x\right]\right]=\Gamma(\mathfrak{g}) \cdot x \text { for all } x \in \mathfrak{g}
$$

and because

$$
\begin{aligned}
a^{j k}\left[e_{i}, e_{j}\right] \wedge\left[e_{i}, e_{k}\right] & =a^{j k} C_{i j}^{m} C_{i k}^{n} e_{m} \wedge e_{n} \\
& =-a^{j k}\left(C_{m n}^{i} C_{j i}^{k}+C_{n j}^{i} C_{m i}^{k}\right) e_{m} \wedge e_{n} \\
& =-a^{j k} C_{m j}^{i} C_{n i}^{k} e_{n} \wedge e_{m} \\
& =-a^{j k} C_{i j}^{m} C_{i k}^{n} e_{m} \wedge e_{n}=0 .
\end{aligned}
$$

\section{REPRESENTATION THEORY AND BI-INVARIANT AFFINE CONNECTIONS ON SIMPLE LIE GROUPS}

The stage is now set for the main result of this paper.

Theorem 8.1. If $G$ is a compact simple Lie group then the space of bi-invariant affine connections on $G$ is one-dimensional in all cases except for $S U(n)$ when $n \geq 3$. In the case of $S U(n)$ there is a two-dimensional family of bi-invariant affine connections. Note that $S O(6)$ behaves the same way as $S U(4)$.

Proof. By Theorem 6.1, the space of bi-invariant affine connections consists of all intertwining maps $\mathfrak{g} \otimes \mathfrak{g} \rightarrow \mathfrak{g}$. Since $\mathfrak{g}$ is irreducible and of real type, Schur's lemma shows that the dimension of the space of such maps is equal to the multiplicity of $\mathfrak{g}$ in $\mathfrak{g} \otimes \mathfrak{g}$ (see [2]). This multiplicity can also be computed after complexifying all the representations - a step which makes the representation theory more convenient. A case by case computation was used to determine the actual multiplicities.

The space $\mathfrak{g} \otimes \mathfrak{g}$ splits into a direct sum $S^{2} \mathfrak{g} \oplus \bigwedge^{2} \mathfrak{g}$ of symmetric and skewsymmetric parts. Table I gives the decomposition of these spaces into irreducible parts. In the table, representations are specified by maximal weights. These weights depend on an ordering of simple roots and the ordering which is used is that which appears in [4]. The results in the table were computed primarily by means of Young-tableaux [3] and dimension counting [1, 9]. Even in the case of the orthogonal groups, the tableau approach was reasonably simple because the adjoint representation is not spinor. The fact that the trivial representation occurs exactly once (and in $S^{2} \mathfrak{g}$ ) was needed in the exceptional cases where dimension counting was used. Finally, Lemma 7.1 was particularly useful for determining the decomposition of $\Lambda^{2} \mathfrak{g}$. The space $\Lambda^{2} \mathfrak{g}$ had to include one copy of $\mathfrak{g}$ (because the natural map $\Lambda^{2} \mathfrak{g} \rightarrow \mathfrak{g}$ is surjective) and the remaining part, although possibly reducible, had to have a specific Casimir operator. These operators could be computed from the techniques in [6] and the tables in [9]. The copy of $\mathfrak{g}$ in $\Lambda^{2} \mathfrak{g}$ corresponds to the affine connections described after Theorem 6.1. The table shows that the only other bi-invariant affine connections occur on $S U(n)$ for $n \geq 3$. 
TABLE I. Decompositions of $S^{2} \mathfrak{g}$ and $\Lambda^{2} \mathfrak{g}$ into irreducible $G$-modules for the compact simple Lie groups. Representations specified by maximal weights

\begin{tabular}{|cccc|}
\hline$G$ & $\mathfrak{g}$ & $S^{2} \mathfrak{g}$ & $\Lambda^{2} \mathfrak{g}$ \\
\hline$A_{1}$ & $\left(2 \lambda_{1}\right)$ & $\left(4 \lambda_{1}\right) \oplus(0)$ & $\left(2 \lambda_{1}\right)$ \\
$A_{2}$ & $\left(\lambda_{1}+\lambda_{2}\right)$ & $\left(2 \lambda_{1}+2 \lambda_{2}\right) \oplus\left(\lambda_{1}+\lambda_{2}\right) \oplus(0)$ & $\left(3 \lambda_{1}\right) \oplus\left(3 \lambda_{2}\right) \oplus\left(\lambda_{1}+\lambda_{2}\right)$ \\
$A_{3}$ & $\left(\lambda_{1}+\lambda_{3}\right)$ & $\left(2 \lambda_{1}+2 \lambda_{3}\right) \oplus\left(2 \lambda_{2}\right) \oplus\left(\lambda_{1}+\lambda_{3}\right) \oplus(0)$ & $\left(2 \lambda_{1}+\lambda_{2}\right) \oplus\left(\lambda_{2}+2 \lambda_{3}\right) \oplus\left(\lambda_{1}+\lambda_{3}\right)$ \\
$A_{n}(n>3)$ & $\left(\lambda_{1}+\lambda_{n}\right)$ & $\left(2 \lambda_{1}+2 \lambda_{n}\right) \oplus\left(\lambda_{2}+\lambda_{n-1}\right) \oplus\left(\lambda_{1}+\lambda_{n}\right) \oplus(0)$ & $\left(2 \lambda_{1}+\lambda_{n-1}\right) \oplus\left(\lambda_{2}+2 \lambda_{n}\right) \oplus\left(\lambda_{1}+\lambda_{n}\right)$ \\
$B_{2}$ & $\left(2 \lambda_{2}\right)$ & $\left(4 \lambda_{2}\right) \oplus\left(2 \lambda_{1}\right) \oplus\left(\lambda_{1}\right) \oplus(0)$ & $\left(\lambda_{1}+2 \lambda_{2}\right) \oplus\left(2 \lambda_{2}\right)$ \\
$B_{3}$ & $\left(\lambda_{2}\right)$ & $\left(2 \lambda_{2}\right) \oplus\left(2 \lambda_{3}\right) \oplus\left(2 \lambda_{1}\right) \oplus(0)$ & $\left(\lambda_{1}+2 \lambda_{3}\right) \oplus\left(\lambda_{2}\right)$ \\
$B_{4}$ & $\left(\lambda_{2}\right)$ & $\left(2 \lambda_{2}\right) \oplus\left(2 \lambda_{4}\right) \oplus\left(2 \lambda_{1}\right) \oplus(0)$ & $\left(\lambda_{1}+\lambda_{3}\right) \oplus\left(\lambda_{2}\right)$ \\
$B_{n}(n>4)$ & $\left(\lambda_{2}\right)$ & $\left(2 \lambda_{2}\right) \oplus\left(\lambda_{4}\right) \oplus\left(2 \lambda_{1}\right) \oplus(0)$ & $\left(\lambda_{1}+\lambda_{3}\right) \oplus\left(\lambda_{2}\right)$ \\
$C_{n}(n>1)$ & $\left(2 \lambda_{1}\right)$ & $\left(4 \lambda_{1}\right) \oplus\left(2 \lambda_{2}\right) \oplus\left(\lambda_{2}\right) \oplus(0)$ & $\left(2 \lambda_{1}+\lambda_{2}\right) \oplus\left(2 \lambda_{1}\right)$ \\
$D_{3}$ & $\left(\lambda_{2}+\lambda_{3}\right)$ & $\left(2 \lambda_{2}+2 \lambda_{3}\right) \oplus\left(2 \lambda_{1}\right) \oplus\left(\lambda_{2}+\lambda_{3}\right) \oplus(0)$ & $\left(\lambda_{1}+2 \lambda_{2}\right) \oplus\left(\lambda_{1}+2 \lambda_{3}\right) \oplus\left(\lambda_{2}+\lambda_{3}\right)$ \\
$D_{4}$ & $\left(\lambda_{2}\right)$ & $\left(2 \lambda_{2}\right) \oplus\left(2 \lambda_{1}\right) \oplus\left(2 \lambda_{3}\right) \oplus\left(2 \lambda_{4}\right) \oplus(0)$ & $\left(\lambda_{1}+\lambda_{3}+\lambda_{4}\right) \oplus\left(\lambda_{2}\right)$ \\
$D_{5}$ & $\left(\lambda_{2}\right)$ & $\left(2 \lambda_{2}\right) \oplus\left(\lambda_{4}+\lambda_{5}\right) \oplus\left(2 \lambda_{1}\right) \oplus(0)$ & $\left(\lambda_{1}+\lambda_{3}\right) \oplus\left(\lambda_{2}\right)$ \\
$D_{n}(n>5)$ & $\left(\lambda_{2}\right)$ & $\left(2 \lambda_{2}\right) \oplus\left(\lambda_{4}\right) \oplus\left(2 \lambda_{1}\right) \oplus(0)$ & $\left(\lambda_{1}+\lambda_{3}\right) \oplus\left(\lambda_{2}\right)$ \\
$E_{6}$ & $\left(\lambda_{2}\right)$ & $\left(2 \lambda_{2}\right) \oplus\left(\lambda_{1}+\lambda_{6}\right) \oplus(0)$ & $\left(\lambda_{4}\right) \oplus\left(\lambda_{2}\right)$ \\
$E_{7}$ & $\left(\lambda_{1}\right)$ & $\left(2 \lambda_{1}\right) \oplus\left(\lambda_{6}\right) \oplus(0)$ & $\left(\lambda_{3}\right) \oplus\left(\lambda_{1}\right)$ \\
$E_{8}$ & $\left(\lambda_{8}\right)$ & $\left(2 \lambda_{8}\right) \oplus\left(\lambda_{1}\right) \oplus(0)$ & $\left(\lambda_{7}\right) \oplus\left(\lambda_{8}\right)$ \\
$F_{4}$ & $\left(\lambda_{1}\right)$ & $\left(2 \lambda_{1}\right) \oplus\left(2 \lambda_{4}\right) \oplus(0)$ & $\left(\lambda_{2}\right) \oplus\left(\lambda_{1}\right)$ \\
$G_{2}$ & $\left(\lambda_{2}\right)$ & $\left(2 \lambda_{2}\right) \oplus\left(2 \lambda_{1}\right) \oplus(0)$ & $\left(3 \lambda_{1}\right) \oplus\left(\lambda_{2}\right)$ \\
\hline
\end{tabular}

\section{BI-INVARIANT AFFINE CONNECTIONS ON COMPACT LIE GROUPS}

The following theorem extends the results in Theorem 8.1 to arbitrary compact Lie groups.

Theorem 9.1. Suppose $G$ is a compact Lie group and suppose the Lie algebra of $G$ splits as $\mathfrak{g}=\mathfrak{z} \oplus \mathfrak{g}_{1} \oplus \cdots \oplus \mathfrak{g}_{q}$, where the center $\mathfrak{z}$ has dimension $p$ and where the $\mathfrak{g}_{i}$ are the simple ideals in $\mathfrak{g}$. Let $r$ be the number of $\mathfrak{s u}(n)$ 's $(n \geq 3)$ in $\mathfrak{g}$. Then the dimension of the space of bi-invariant affine connections on $G$ is given by

$$
\operatorname{dim}\left(\mathscr{A}_{G \times G} \operatorname{Fr}(G)\right)=p^{3}+3 \cdot p \cdot q+q+r .
$$

Proof. Irreducible representations of $\mathfrak{g}$ which are trivial on $\mathfrak{z}$ can be expressed as $\left(0, \rho_{1}, \ldots, \rho_{q}\right)$. This represents the tensor product of the trivial representation of $\mathfrak{z}$ with representations $\rho_{i}$ of $\mathfrak{g}_{i}$ for $i=1, \ldots, q$. In this notation

$$
\mathfrak{g}=p \cdot(0,0, \ldots, 0) \oplus \bigoplus_{i=1}^{q}\left(0,0, \ldots, \mathfrak{g}_{i}, \ldots, 0\right) .
$$

Computing $\mathfrak{g} \otimes \mathfrak{g}$ gives 


$$
\begin{array}{cc}
\mathfrak{g} \otimes \mathfrak{g}= & p^{2} \cdot(0,0, \ldots, 0) \\
\oplus \bigoplus_{i=1}^{q} & 2 \cdot p \cdot\left(0,0, \ldots, \mathfrak{g}_{i}, \ldots, 0\right) \\
\oplus \bigoplus_{i=1}^{q} & \left(0,0, \ldots, \mathfrak{g}_{i} \otimes \mathfrak{g}_{i}, \ldots, 0\right) \\
\oplus \bigoplus_{i<j} 2 \cdot\left(0,0, \ldots, \mathfrak{g}_{i}, \ldots, \mathfrak{g}_{j}, \ldots, 0\right) .
\end{array}
$$

The first line of this expression results in $p^{3}$ independent intertwining maps $\mathfrak{g} \otimes \mathfrak{g} \rightarrow \mathfrak{g}$. The second line gives $2 \cdot p \cdot q$ more. The third line gives $p \cdot q$ maps to the center part of $\mathfrak{g}$ and, by Theorem 8.1, $q+r$ more maps to the simple parts of $\mathfrak{g}$. Finally, there are no such maps between the representations in the fourth line and $\mathfrak{g}$.

\section{AFFINE CONNECTIONS ON $S U(n)$ AND $U(n)$}

Theorem 9.1 shows that the Lie group $U(n)$ has a six-dimensional family of bi-invariant affine connections. These connections are described more precisely in the following theorem.

Theorem 10.1. In terms of bilinear maps $\eta: \mathfrak{u}(n) \times \mathfrak{u}(n) \rightarrow \mathfrak{u}(n)$, a basis for the six-dimensional space of bi-invariant affine connections on $U(n) \quad(n \geq 3)$ is given by

$$
\begin{array}{ll}
\eta_{1}(x, y)=x \cdot y-y \cdot x, & \eta_{2}(x, y)=i \cdot(x \cdot y+y \cdot x), \\
\eta_{3}(x, y)=i \cdot \operatorname{tr}(x) \cdot y, & \eta_{4}(x, y)=i \cdot \operatorname{tr}(y) \cdot x, \\
\eta_{5}(x, y)=\operatorname{tr}(x \cdot y) \cdot i I, & \eta_{6}(x, y)=\operatorname{tr}(x) \cdot \operatorname{tr}(y) \cdot i I .
\end{array}
$$

Here, $I$ is the $n \times n$ identity matrix.

Proof. First, it is straightforward to verify that the $\eta_{j}$ are all bilinear and that they preserve $\mathfrak{u}(n)$. In addition, they all satisfy the condition $\eta\left(\operatorname{Ad}_{g} x, \operatorname{Ad}_{g} y\right)$ $=\operatorname{Ad}_{g} \eta(x, y)$. Since Theorem 9.1 shows that the space of bi-invariant affine connections is six-dimensional $(p=q=r=1)$, all that needs to be proven is that the $\eta_{j}$ are linearly independent. Suppose $\sum a_{j} \eta_{j}(x, y)=0 \quad \forall x, y \in \mathfrak{g}$. Let $E_{j k}$ be the matrix with a 1 in the $(j, k)$-position and 0 's elsewhere. Letting $x=i \cdot E_{11}-i \cdot E_{22}$ and $y=i \cdot E_{22}-i \cdot E_{33}$ gives $a_{2}=a_{5}=0$. Next, by letting $x=i \cdot E_{11}$ and $y=i \cdot E_{22}$ it follows that $a_{3}=a_{4}=a_{6}=0$. Finally, the values $x=E_{12}-E_{21}$ and $y=i \cdot E_{12}+i \cdot E_{21}$ imply $a_{1}=0$.

Note that $U(2)$ only has a five-dimensional family of bi-invariant affine connections. The linear dependence of the $\eta_{j}$ in the case $n=2$ corresponds to the following identity in $\mathfrak{g l}(2, \mathbb{C})$ :

$$
(x \cdot y+y \cdot x)=\operatorname{tr}(x) \cdot y+\operatorname{tr}(y) \cdot x+\operatorname{tr}(x \cdot y) \cdot I-\operatorname{tr}(x) \cdot \operatorname{tr}(y) \cdot I .
$$

By Theorem 8.1 the Lie group $S U(n)$ has a two-dimensional family of biinvariant affine connections. As with $U(n)$ it is simple to verify that these connections are given by

$$
\eta(x, y)=\mu \cdot[x, y]+\nu \cdot\left(i \cdot(x \cdot y+y \cdot x)-\frac{2}{n} \cdot \operatorname{tr}(x \cdot y) \cdot i I\right)
$$

mapping $\mathfrak{s u}(n) \times \mathfrak{s u}(n) \rightarrow \mathfrak{s u}(n)$ for arbitrary real numbers $\mu$ and $\nu$. 


\section{REFERENCES}

1. M. R. Bremner, R. V. Moody, and J. Patera, Tables of dominant weight multiplicities for representations of simple lie algebras, Marcel Dekker, New York, 1985.

2. T. Bröcker and T. tom Dieck, Representations of compact Lie groups, Springer-Verlag, New York, 1985.

3. M. Fischler, Young-tableau methods for Kronecker products of representations of the classical groups, J. Math. Phys. 22 (1981), 637-648.

4. S. Helgason, Differential geometry, Lie groups, and symmetric spaces, Academic Press, New York, 1978.

5. S. Kobayashi and K. Nomizu, Foundations of differential geometry, Interscience, New York, 1963, 1969.

6. H. T. Laquer, Stability properties of the Yang-Mills functional near the canonical connection, Michigan Math. J. 31 (1984), 139-159.

7. Invariant gauge automorphisms of homogeneous principal bundles, Geom. Dedicata 33 (1990), 27-35.

8. __ Invariant affine connections on symmetric spaces, Proc. Amer. Math. Soc. (to appear).

9. W. G. McKay and J. Patera, Tables of dimensions, indices, and branching rules for representations of simple Lie algebras, Marcel Dekker, New York, 1981.

10. K. Nomizu, Invariant affine connections on homogeneous spaces, Amer. J. Math. 76 (1954), 33-65.

11. H. C. Wang, On invariant connections over a principal fibre bundle, Nagoya Math. J. 13 (1958), 1-19.

Department of Mathematics, Idaho State University, Pocatello, Idaho 83209 